\title{
OSTEOARTHRITIS
}

\section{Complement-mediated inflammation in OA progression}

The inflammatory complement cascade has a key role in the pathogenesis of osteoarthritis (OA), according to a Nature Medicine paper from William Robinson and colleagues at Stanford University School of Medicine, USA.

"OA has long been viewed to arise from 'wear and tear' of the cartilage and joint, and although it affects over a third of people over 60 years of age there are currently no disease-slowing drugs available," explains Robinson. This situation prompted their research into the role of inflammation in the pathogenesis of OA.

First, the investigators performed a synovial fluid proteomic screen: complement proteins were found to be overexpressed in OA synovial fluid in comparison with fluid from healthy donors. A synovial membrane transcriptome analysis was then carried out: levels of complement effector transcripts were markedly higher and levels of complement inhibitor transcripts were considerably lower in synovial membranes from patients with OA than from healthy donors. These findings indicate that complement expression and activation in the synovium has a role in this disease.

"Next we took a genetic and pharmacologic approach to investigate the role of the complement system in

mouse models of OA," says

Robinson. Mice unable to secrete C5 (a central complement effector) or possessing dysfunctional C6 (a component of the membrane attack complex [MAC]) were protected against development of OA following medial meniscectomy, whereas mice deficient for the MAC-inhibitor CD59a developed more severe disease than wild-

type littermates. Disease initiated by destabilization of the medial meniscus (DMM) or that developed spontaneously due to increasing age were also more severe in mice deficient for CD59a in comparison with wild-type mice. These findings in genetically modified mice, implicating the MAC as a mediator of damage in $\mathrm{OA}$, were confirmed using pharmacological agents.

Finally, the authors investigated how the MAC might lead to cartilage damage, and showed that, rather than leading to chondrocyte lysis, in this context the MAC sublytically stimulates chondrocytes to express genes that have been previously implicated in OA progression, including those encoding cartilage-degrading enzymes and proinflammatory cytokines.
"In our ongoing research we are further investigating how the complement system is activated in $\mathrm{OA}$ joints, and how formation of the MAC stimulates production of inflammatory and degradative mediators", explains Robinson. He concludes that, "although there are prior studies from the 1990's showing deposition of MAC on OA cartilage, the extensive dysregulation of expression of complement and its role in OA pathogenesis was not previously appreciated."

Jenny Buckland
Original article Wang, Q. et al. Identification of a central role for complement in osteoarthritis. Nat. Med. doi: $10.1038 / \mathrm{nm} .2543$ 